\title{
Taking a back seat: synaptic vesicle clustering in presynaptic terminals
}

\author{
Arndt Pechstein ${ }^{1,2}$ and Oleg Shupliakov ${ }^{1 *}$ \\ Department of Neuroscience, Developmental Biology for Regenerative Medicine, Karolinska Institutet, Stockholm, Sweden \\ Department of Membrane Biochemistry, Institute of Chemistry and Biochemistry, Freie Universität Berlin, Germany
}

Edited by:

Robert Renden, UCB Pharma SA,

Belgium

Reviewed by:

Serge Marty, Ecole Normale

Supérieure, France

Thomas G. Oertner, Friedrich Miescher

Institute for Biomedical Research,

Switzerland

*Correspondence:

Oleg Shupliakov, Karolinska Institutet, Department of Neuroscience,

Developmental Biology for

Regenerative Medicine, Von Eulers väg

3, 17177 Stockholm, Sweden.

e-mail: oleg.shupliakov@ki.se
Central inter-neuronal synapses employ various molecular mechanisms to sustain neurotransmitter release during phases of high-frequency synaptic activity. One of the features ensuring this property is the presence of a pool of synaptic vesicles (SVs) in the presynaptic terminal. At rest and low rates of stimulation, most of the vesicles composing this pool remain in a tight cluster. They are actively utilized when neurons fire action potentials at higher rates and the capability of the recycling machinery is limited. In addition, SV clusters are capable of migrating between release sites and reassemble into clusters at neighboring active zones (AZs). Within the cluster, thin "tethers" interconnect SVs. These dynamic filamentous structures are reorganized during stimulation thereby releasing SVs from the cluster. So far, one protein family, the synapsins, which bind actin filaments and vesicles in a phosphorylation-dependent manner, has been implicated in SV clustering in vertebrate synapses. As evident from recent studies, many endocytic proteins reside in the SV cluster in addition to synapsin. Here we discuss alternative possible mechanisms involved in the organization of this population of SVs. We propose a model in which synapsins together with other synaptic proteins, a large proportion of which is involved in SV recycling, form a dynamic proteinaceous "matrix" which limits the mobility of SVs. Actin filaments, however, do not seem to contribute to SV crosslinking within the SV cluster, but instead they are present peripherally to it, at sites of neurotransmitter release, and at sites of SV recycling.

Keywords: synapse, synaptic vesicle cluster, scaffolding proteins, endocytosis, active zone, periactive zone, actin, synapsin

\section{INTRODUCTION}

Despite the existence of different types of synapses in a variety of organisms all presynaptic terminals share a similar structural organization. For neurons to fulfill their common task that is converting an electrical into a chemical signal, synaptic transmission has to be extremely fast. A high level of structural organization is the basis for their remarkable features. As a prerequisite for the quantal release of neurotransmitters following depolarization, synapses harbor a varying number of synaptic vesicles (SVs) accumulated around release sites. These SVs undergo continuous cycles within the axonal bouton. While a fair amount of knowledge has been gained on some aspects of the SV cycle, such as exo- and endocytosis, others, such as SV trafficking and clustering are poorly understood. In particular, the molecular basis for SV clustering remains largely enigmatic. By highlighting known functions of proteins located within the SV cluster we discuss in this review putative mechanisms by which SVs might be held together in a cluster thereby controlling SV mobility and availability.

\section{TAKING A REST: RESTING VESICLES IN CENTRAL SYNAPSES ARE ORGANIZED IN A CLUSTER}

Although SVs all look alike in an electron microscope they greatly differ with regard to their behavior following depolarization. This observation led to the creation of the pool concept which classifies SVs according to their release probability and position with respect to the active zone (AZ). Depending on whether morphological or physiological/functional criteria were considered the presynaptic collection of SVs has been subdivided into distinct pools. Physiologically, SVs are distinguished based on their release probability as the readily releasable pool (RRP; those closest to the AZ which are released first) (Schikorski and Stevens, 2001) and the reserve pool (RP; a by far higher number of SVs situated more distal to the release site) which replenishes the RRP upon its depletion (Südhof, 2004). The RRP and the RP together constitute the recycling pool defined as SVs being able to run through exo/endocytic cycles during prolonged stimulation (Cochilla et al., 1999; Schikorski and Stevens, 2001). Since the total recycling pool accounts for only about $5-20 \%$ of the total SVs in some synapses, the existence of a third pool has been suggested (resting pool) not actively contributing to neurotransmission under normal conditions, but only upon intense stimulation (Südhof, 2000; Rizzoli and Betz, 2005; Fdez and Hilfiker, 2006). Morphologically, SVs have been subdivided into those few being very close to the membrane (docked vesicles) and those further away than 150-200 nm (reserve pool) accounting for the majority of all SVs (Pieribone et al., 1995; Südhof, 2000; Fdez and Hilfiker, 2006). Thus, currently there seems to be no general consensus in the field about the classification of physiologically and morphologically defined SV pools. Furthermore, vesicles in many synapses appear to be not anatomically segregated but mixed and interchanged between the different pools (Schikorski and Stevens, 
2001; Rizzoli and Betz, 2005). Finally, the number and size of vesicle pools defined physiologically and morphologically vary considerably between organisms and synapses with different release properties which makes classifications even more complex (Brodin et al., 1999; Richards et al., 2003).

A recurring feature, however, is the organization of distal SVs in clusters or tight aggregations at rest. These clusters may vary in size and are particularly distinct in some "phasic" central synapses which most of the time are silent or release neurotransmitters at low rate and may sustain neurotransmitter release during phases of highfrequency synaptic activity. An example of such an inter-neuronal junction is the excitatory reticulospinal synapse established by command neurons, which activates the locomotor generator in the spinal cord. The presence of tight clusters of SVs is a common feature of these inter-neuronal junctions in different vertebrate species (Li et al., 1995; Pieribone et al., 1995). Therefore, to highlight the general concept of SV clustering irrespective of organismal or cell type-specific constraints we refer to the SV cluster as those SVs which are distal to the sites of release (more than 150-200 nm away from the presynaptic membrane), interconnected via filamentous tethers (see below), and different in mobility and release probability from those immediately adjacent to the AZ.

\section{DANGLING ON A STRING: FILAMENTOUS TETHERS INTERCONNECT SVS IN THE CLUSTER}

Quick freeze - deep etch ultrastructural analysis and electron tomography studies revealed that SVs in clusters are linked to each other by thin tethers (Hirokawa et al., 1989; Gustafsson et al., 2002; Siksou et al., 2007; Fernandez-Busnadiego et al., 2010) (Figure 1) which were originally thought to be composed of synapsin I (Hirokawa et al., 1989). In this regard, many studies have focused on the synapsin family of peripheral vesicle proteins that have been suggested to maintain SVs in the cluster linking them to actin filaments (Greengard et al., 1993; Pieribone et al., 1995; Evergren et al., 2007a) and which upon depolarization and $\mathrm{Ca}^{2+}$ influx are phosphorylated thereby releasing SV s from the F-actin matrix to the RRP (Chi et al., 2001; Chi et al., 2003).

The link between synapsin and F-actin and the suggestion of a role of this interaction in SV clustering emerged from studies demonstrating that synapsin (i) has actin-nucleating properties (Valtorta, 1992), (ii) can promote filamentous actin (F-actin) polymerization (Fesce et al., 1992), (iii) stabilizes and bundles F-actin (Bähler and Greengard, 1987), and that (iv) free and SV-bound synapsin may interact with F-actin in a phosphorylation-dependent manner (Benfenati et al., 1992). However, while there is a strong immunoreactivity for synapsin in the SV cluster in the majority of central synapses (Pieribone et al., 1995; Bloom et al., 2003; Tao-Cheng, 2006) direct evidence for the presence of actin filaments in this region and hence a direct role of actin in SV clustering is lacking. Contrary, several studies instead revealed that actin filaments are surrounding SV clusters and are present at AZs in lamprey (Bloom et al., 2003; Bourne et al., 2006) and rat hippocampal neurons (Sankaranarayanan et al., 2003) (Figure 2D). Moreover, preventing actin polymerization by application of latrunculin or swinholide to lamprey reticulospinal synapses (Shupliakov et al., 2002; Bourne et al., 2006), frog NMJs (Gaffield et al., 2006), and mouse NMJs (Gaffield and Betz, 2007) did not change the organization of SV

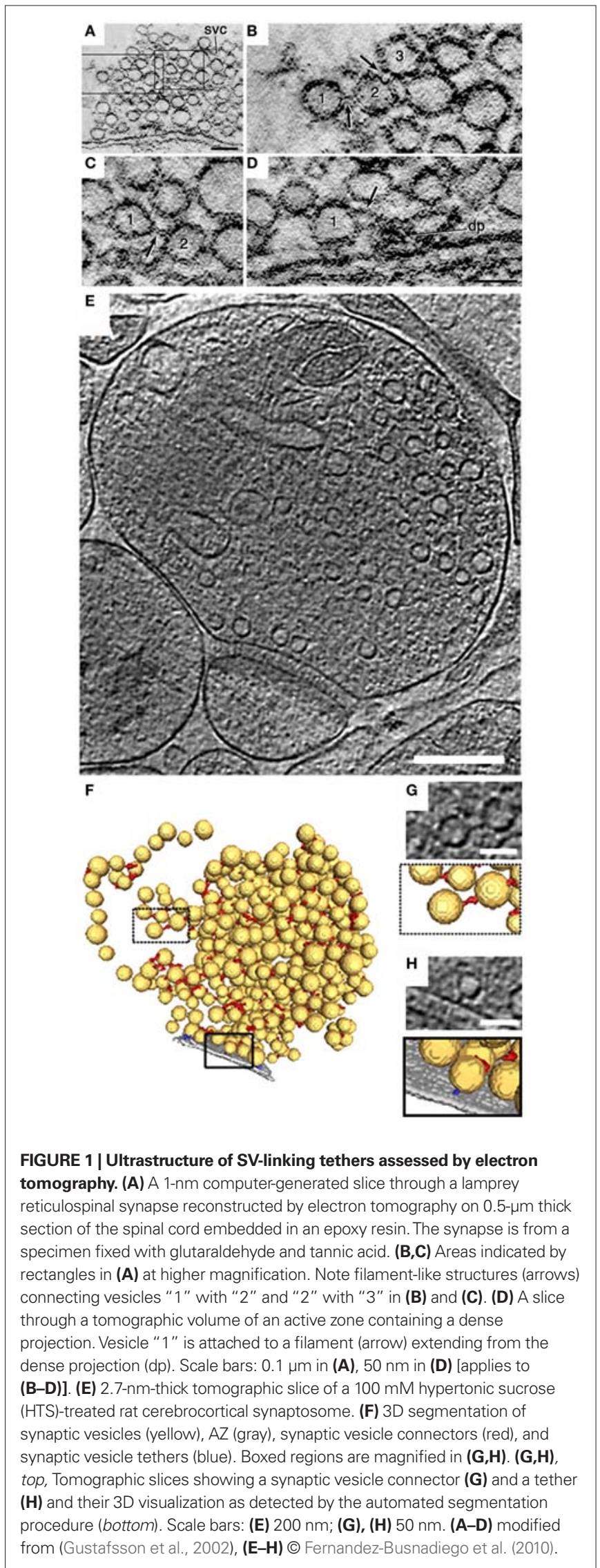



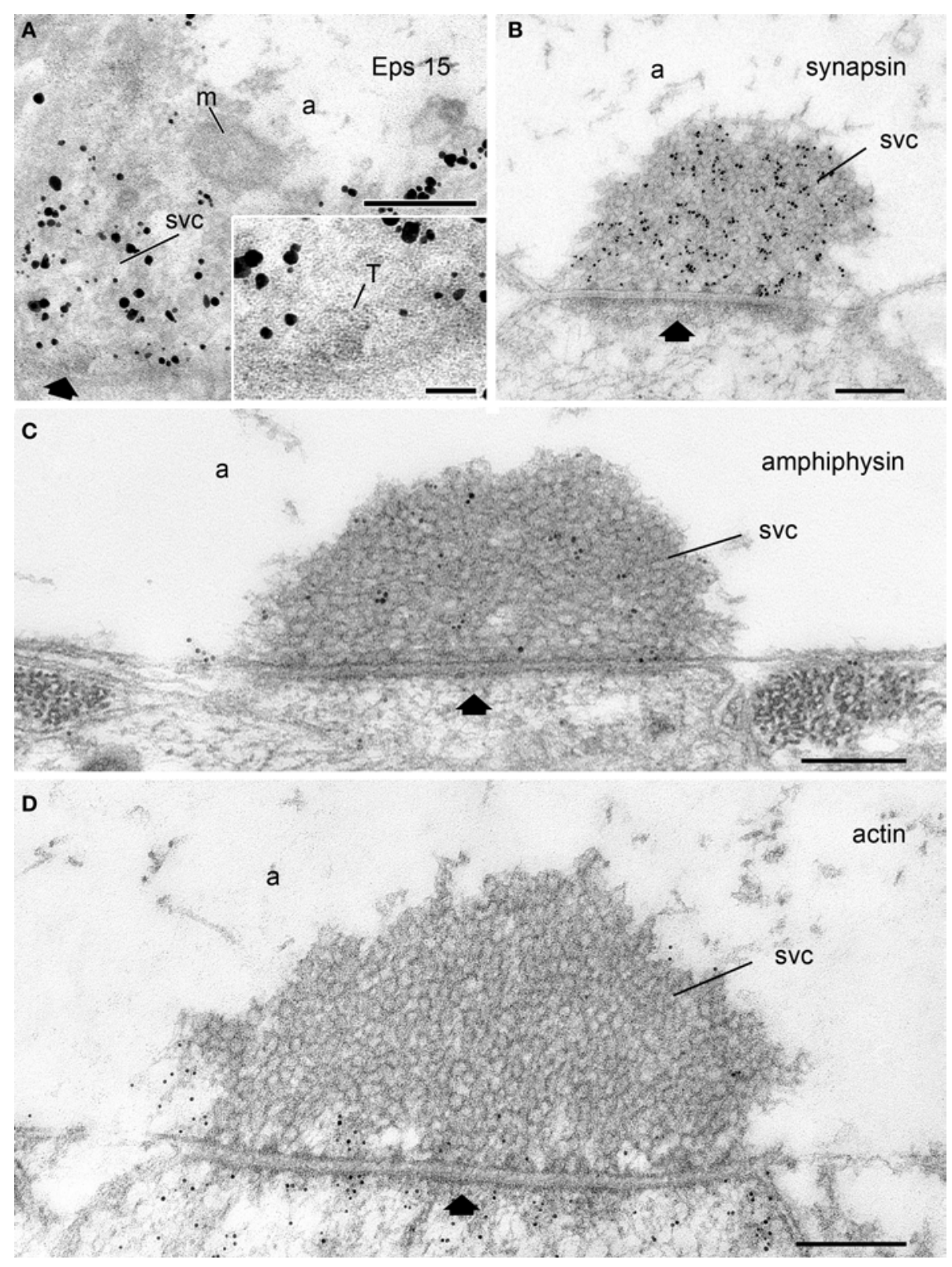

FIGURE 2 | Subcellular localization of synapsin, actin, and the endocytic proteins Eps 15 and amphiphysin at rest with respect to the SV cluster as visualized by electron microscopy. (A) Eps15 immunoreactivity is concentrated over the SV cluster in Drosophila neuromuscular junctions (NMJs) as revealed by immunogold labeling. Inset: A region of the SV cluster (SVC) containing a cross-section of a T-bar (T) is shown at higher magnification. Unpublished image from Shupliakov's group from the data set analyzed and published in (Koh et al., 2007). (B-D) Electron micrographs of lamprey giant reticulospinal synapses at rest labeled with antibodies against synapsin (B), amphiphysin (C), or F-actin (D). Unpublished images from Shupliakov's group from the data sets analyzed and published in (Bloom et al., 2003; Evergren et al., 2004). Note the difference in localization of the proteins. While synapsin and amphiphysin labeling is concentrated over the SV cluster (SVC) in (B,C), a weak actin labeling is present only around the SV cluster and at the active zone (black arrow), and at the presynaptic membrane peripheral to the SV cluster. The vesicle cluster is devoid of gold particles (D). a, axoplasm; m, mitochondrium; SVC, synaptic vesicle cluster; black arrow, active zone. Scale bars: A-D: $200 \mathrm{~nm}$; inset in A: $50 \mathrm{~nm}$. clusters in these synapses or the mobility of SVs therein. Several studies indicated that actin might have a scaffolding role in retaining regulatory molecules including synapsin at synaptic terminals and guide them to their functional destination during repeated cycles of stimulation (Shupliakov et al., 2002; Sankaranarayanan et al., 2003). Consistent with this idea, a profound actin-rich cytomatrix was found surrounding the SV cluster in the periactive zone (Shupliakov et al., 2002; Bloom et al., 2003; Sankaranarayanan et al., 2003). Thus, the scarcity of actin filaments within the SV cluster indicates that SVs are tethered by different means.

Synapsins may dimerize and may thus crosslink vesicles in a cluster in the absence of actin filaments (Monaldi et al., 2009). Genetic studies speak in favor for this possibility. It has been shown that the knockout $(\mathrm{KO})$ of synapsin genes leads to a reduction in the number 
of SVs in nerve terminals (Li et al., 1995; Ryan et al., 1996; Terada et al., 1999; Gitler et al., 2004). However, SVs in those synapses are still aggregating at release sites suggesting that synapsins are not the only molecules which may hold vesicles together. All aspects of SV mobility are indistinguishable between WT and synapsin triple KO mice (Gaffield and Betz, 2007) and filamentous tethers linking SVs to each other are also present in synapsin triple $\mathrm{KO}$ mice (Siksou et al., 2007). In Drosophila neuromuscular junctions, genetic deletion of synapsin does not cause significant changes in the vesicle organization at AZs at rest (Godenschwege et al., 2004). Moreover, in synapses lacking synapsins, e.g., ribbon synapses, filamentous structures crosslinking vesicles are also present (Usukura and Yamada, 1987; Lenzi et al., 1999; Parsons and Sterling, 2003). Finally, transgenic expression of synapsin in photoreceptor cells, which usually do not express this protein, did not affect the structure or physiology of these synapses (Geppert et al., 1994).

Hence, neither the synapsin-tethered vesicle-to-vesicle model nor the synapsin-actin filament-vesicle model fully explains the mechanisms by which SVs are organized in the resting pool. It is therefore reasonable to assume that synapsin is just one of the molecules participating in SV clustering. The major structural change observed in synapsin I KO mice is a decrease in the packing density of SVs, thus suggesting that synapsins which may form homo- or heterodimers are particularly involved in the formation of extremely densely packed clusters such as those present in phasic reticulospinal synapses at rest for instance. Interestingly, almost ideal hexagonal packing correlates with the presence of a dense synapsin immunoreactivity within the SV cluster in these synapses (Figure 2B).

\section{THE UNKNOWN PUPPET MASTER: OTHER PROTEINS CONTROLLING SV MOBILITY}

What other components may contribute to the universal process of SV clustering? A remarkable yet still ill-understood feature revealed in central synapses and in Drosophila NMJs is an accumulation of key endocytic molecules in the SV cluster under resting conditions. Amongst them are large endocytic proteins such as intersectin 1/Dap160 (Koh et al., 2004, 2007; Evergren et al., 2007b) as well as the dephosphins, a group of endocytic proteins including amphiphysin, dynamin, epsin, synaptojanin, endophilin, and Eps15 (Haffner et al., 1997; Evergren et al., 2004, 2007b; Koh et al., 2007; Jakobsson et al., 2008) (Figures 2A,C) which become dephosphorylated following $\mathrm{Ca}^{2+}$ influx into the nerve terminal (Cousin et al., 2001). These proteins undergo activity-dependent cycles between the SV pool and the periactive zone (Shupliakov, 2009). An accumulation of them in the SV cluster at rest suggests that they may be part of a proteinaceous matrix which limits the free migration of SVs in the axoplasm (Shupliakov, 2009). Thus, $\mathrm{Ca}^{2+}$ influx and dephosphorylationtriggered migration away from the SV cluster may not only initiate endocytosis but also destabilize the cytoskeletal matrix making SVs accessible for release. Interestingly, proteins that undergo stimulation-dependent migration cycles, including dynamin, intersectin, and amphiphysin, show differential levels in tonic and phasic synapses (Evergren et al., 2006) suggesting that the organization of the SV-surrounding proteinaceous matrix may differ in synapses with different release patterns.

\section{LEAVING AND ENTERING THE SV CLUSTER}

Given that repeated phosphorylation/dephosphorylation cycles, mediated by the antagonistic actions of kinases and phosphatases, play a crucial role in protein targeting and possibly also in SV cluster stabilization, a model in which these modifying enzymes are compartmentalized is tempting. In fact, SV clustering critically depends on protein phosphorylation. The phosphatase inhibitor okadaic acid disrupts SV clusters (Betz and Henkel, 1994; Henkel et al., 1996) and increases vesicle mobility in both vertebrate NMJs (Gaffield et al., 2006; Gaffield and Betz, 2007) and hippocampal neurons (Kraszewski et al., 1996). Likewise, blocking kinases with staurosporine decreases SV mobility (Gaffield and Betz, 2007). Interestingly, several protein kinases and phosphatases associated with synapsin or dephosphin phosphorylation and dephosphorylation, respectively, exhibit a selective distribution. The calcium calmodulin-dependent protein kinase II (CaMKII), for instance, surrounds SV clusters at rest and is absent from the inside of the cluster. Following depolarization, CaMKII disperses and mingles with SVs which start to decluster (Tao-Cheng et al., 2006). This suggests a mechanism in which under resting conditions CaMKII has limited access to synapsin I and possible other target proteins within the SV cluster while depolarization triggers a CaMKIImediated declustering process from the periphery toward the center where the depth of penetration into the SV cluster depends on the duration of CaMKII activation (Tao-Cheng et al., 2006). Similar mechanisms may apply for other kinases known to target synapsin such as mitogen-activated protein (MAP) kinase, c-Src, or protein kinase A (PKA) as well as for dephosphin-modifying enzymes including cyclin-dependent kinase 5 (cdk5) and the phosphatase calcineurin. Thus, a complex proteinaceous network consisting of endocytic proteins undergoing migration cycles regulated by reversible phosphorylation events could constitute a matrix that limits the diffusion of SVs in the SV reserve pool.

Vesicles become mobile not only following synaptic activity during which they leave the cluster, fuse with the presynaptic membrane, and recycle locally using clathrin and bulk endocytosis (Figure 3). Recent studies have shown that SVs in central synapses may also migrate from one release site to another, thus indicating that they integrate into existing vesicle clusters at neighboring AZs. Using fluorescence imaging, correlative electron microscopy, and modeling of vesicle dynamics, Goda and collaborators have shown that some vesicles at synapses form part of a larger vesicle "superpool" (Staras et al., 2010). The vesicles within this "superpool" are highly mobile and are rapidly exchanged between terminals (turnover: approximately $4 \%$ of total $\mathrm{pool} / \mathrm{min}$ ), significantly changing vesicular composition at synapses over time. A local TrkB-receptor-dependent mechanism has been proposed for synapse-specific regulation of presynaptic vesicle pools through control of vesicle release and capture to or from the extrasynaptic pool. Thus, a number of yet unknown additional molecules may be present in the vesicle cluster controlling the entry of vesicles migrating between AZs.

\section{AN ALTERNATIVE MODEL FOR VESICLE CLUSTERING}

Based on the studies discussed above we propose a model in which several presynaptic proteins which are selectively localized to the SV cluster at rest form a complex proteinaceous network forming 
an inter-vesicular matrix (IVM) (Figure 4). This IVM restricts vesicle mobility and accumulates SVs in clusters close to release sites. Its dynamic nature allows for stimulation-triggered assemblydisassembly cycles driven by phosphorylation/dephosphorylation events ensuring regulated neurotransmitter release. In addition, the IVM allows the release and re-entry of vesicles migrating between neighboring AZs. In fact, recent structural studies support this view: a tiny electron-dense matrix distinct from tethers underlying the

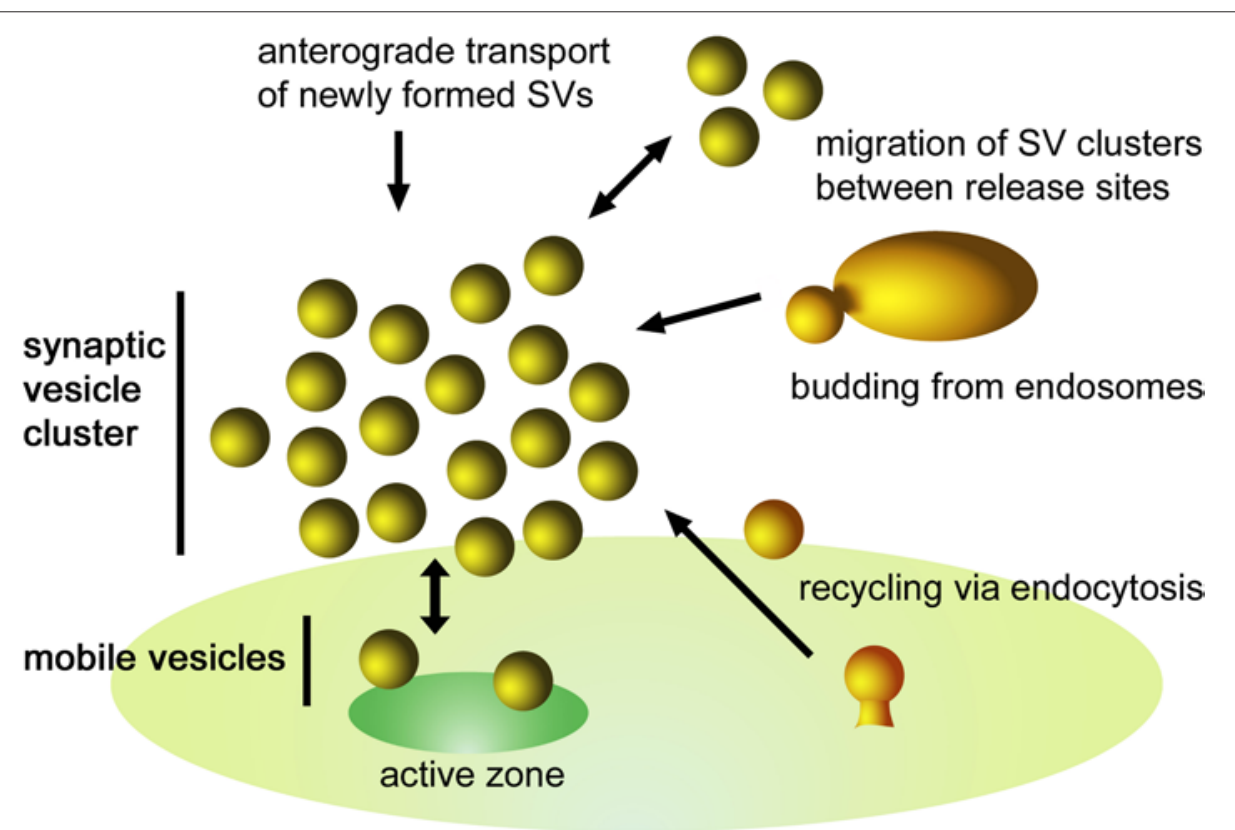

FIGURE 3 | Schematic illustration of the dynamics of a SV cluster. SVs from different sources can be incorporated into a cluster. They may originate from (i) endocytic recycling at the periactive zone, (ii) budding processes from endosomes, (iii) exchange of SV clusters between adjacent release sites, and (iv)

anterograde transport of newly formed SVs. Following depolarization, SVs of the readily releasable pool fuse with the plasma membrane inducing a net flow of SVs toward the active zone. To maintain an equilibrium there is a continuous exchange between the mobile SVs and the SV cluster.

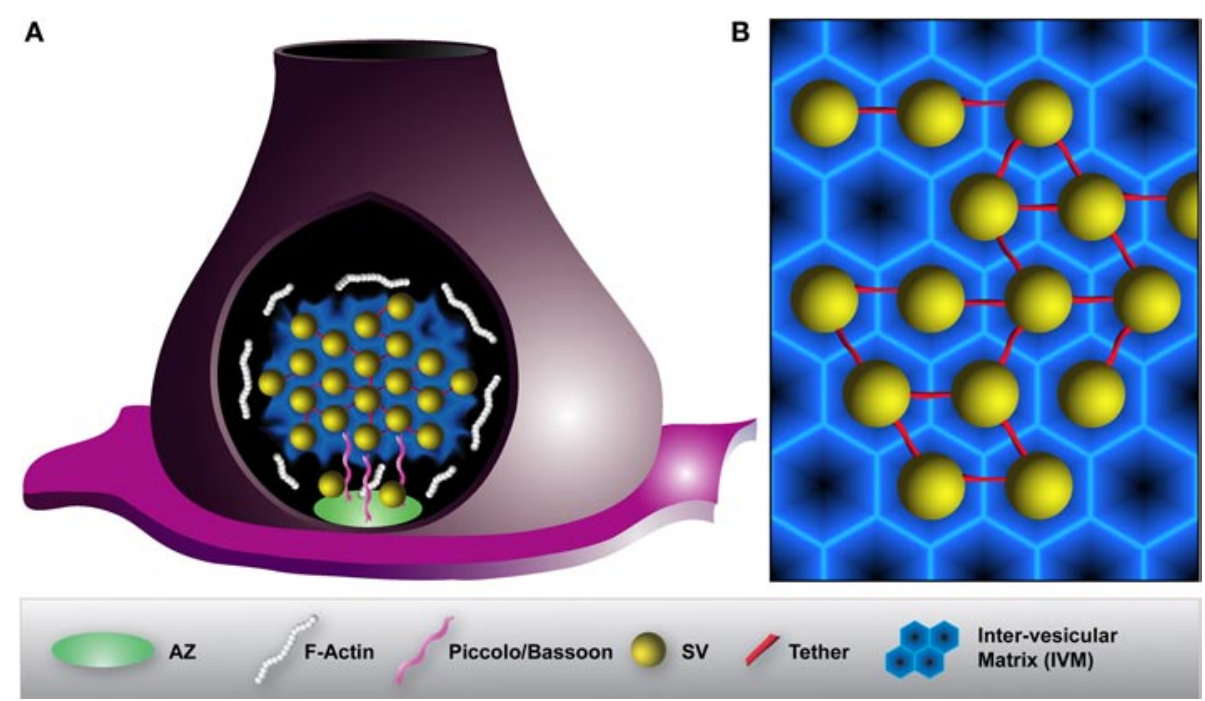

FIGURE 4 | Model illustrating the organization of a SV cluster within a presynaptic terminal. (A) To prevent diffusion of SVs and to maintain them in the SV cluster, vesicles are interconnected by thin tethers (red) and embedded into a proteinaceous inter-vesicular matrix (IVM) (blue). F-actin (white) peripherally surrounds SV clusters but is absent from the inside. Large scaffolding proteins such as Piccolo or Bassoon (pink) projecting from the active zone (AZ) (green) assist the organization and the recruitment of SVs to release sites. (B) Magnification of SV organization within a SV cluster. A hexagonally organized IVM constituted of various synaptic and endocytic proteins may allow for an optimal packing density of SVs. In that way, SVs are encaged to prevent their dispersion. 
vesicle pool was detected in recent tomographic studies (Siksou et al., 2007; Fernandez-Busnadiego et al., 2010). An unresolved question remains pertaining to the molecular composition and organization of the IVM. Furthermore, it remains to be clarified how the IVM is assembled and disassembled in a dynamic fashion, how it regulates the release of SVs to the release site, and finally how SVs are reclustered after retrieval from different sources. This has to be a matter of further studies.

\section{REFERENCES}

Bähler, M., and Greengard, P. (1987). Synapsin I bundles F-actin in a phosphorylation-dependent manner. Nature 326, 704-707.

Benfenati, F., Valtorta, F., Chieregatti, E., and Greengard, P. (1992). Interaction of free and synaptic vesicle-bound synapsin I with F-actin. Neuron 8, 377-386.

Betz, W. J., and Henkel, A. W. (1994). Okadaic acid disrupts clusters of synaptic vesicles in frog motor nerve terminals. J. Cell Biol. 124, 843-854.

Bloom, O., Evergren, E., Tomilin, N., Kjaerulff, O., Low, P., Brodin, L., Pieribone, V. A., Greengard, P., and Shupliakov, O. (2003). Colocalization of synapsin and actin during synaptic vesicle recycling. J. Cell Biol. 161, 737-747.

Bourne, J., Morgan, J. R., and Pieribone, V. A. (2006). Actin polymerization regulates clathrin coat maturation during early stages of synaptic vesicle recycling at lamprey synapses. J. Comp. Neurol. 497, 600-609.

Brodin, L., Bakeeva, L., and Shupliakov, O. (1999). Presynaptic mitochondria and the temporal pattern of neurotransmitter release. Philos. Trans. R. Soc. Lond., B, Biol. Sci. 354, 365-372.

Chi, P., Greengard, P., and Ryan, T. A. (2001). Synapsin dispersion and reclustering during synaptic activity. Nat. Neurosci. 4, 1187-1193.

Chi, P., Greengard, P., and Ryan, T. A. (2003). Synaptic vesicle mobilization is regulated by distinct synapsin I phosphorylation pathways at different frequencies. Neuron 38, 69-78.

Cochilla, A. J., Angleson, J. K., and Betz, W. J. (1999). Monitoring secretory membrane with FM1-43 fluorescence. Annu. Rev. Neurosci. 22, $1-10$.

Cousin, M. A., Tan, T. C., and Robinson, P. J. (2001). Protein phosphorylation is required for endocytosis in nerve terminals: potential role for the dephosphins dynamin I and synaptojanin, but not AP180 or amphiphysin. J. Neurochem. 76, 105-116.

Evergren, E., Benfenati, F., and Shupliakov, O. (2007a). The synapsin cycle: a view from the synaptic endocytic zone. J. Neurosci. Res. 85, 2648-2656.

Evergren, E., Gad, H., Walther, K., Sundborger, A., Tomilin, N., and Shupliakov, O. (2007b). Intersectin is a negative regulator of dynamin recruitment to the synaptic endocytic zone in the central synapse. J. Neurosci. 27, 379-390.

Evergren, E., Marcucci, M., Tomilin, N., Low, P., Slepnev, V., Andersson, F., Gad, H., Brodin, L., De Camilli, P., and Shupliakov, O. (2004). Amphiphysin is a component of clathrin coats formed during synaptic vesicle recycling at the lamprey giant synapse. Traffic 5, 514-528.

Evergren, E., Zotova, E., Brodin, L., and Shupliakov, O. (2006). Differential efficiency of the endocytic machinery in tonic and phasic synapses. Neuroscience 141, 123-131.

Fdez, E., and Hilfiker, S. (2006). Vesicle pools and synapsins: new insights into old enigmas. Brain Cell Biol. 35, 107-115.

Fernandez-Busnadiego, R., Zuber, B., Maurer, U. E., Cyrklaff, M., Baumeister, W., and Lucic, V. (2010). Quantitative analysis of the native presynaptic cytomatrix by cryoelectron tomography. J. Cell Biol. 188, 145-156.

Fesce, R., Benfenati, F., Greengard, P., and Valtorta, F. (1992). Effects of the neuronal phosphoprotein synapsin I on actin polymerization. II. Analytical interpretation of kinetic curves. J. Biol. Chem. 267, 11289-11299.

Gaffield, M. A., and Betz, W. J. (2007). Synaptic vesicle mobility in mouse motor nerve terminals with and without synapsin. J. Neurosci. 27, 13691-13700.

Gaffield, M. A., Rizzoli, S. O., and Betz, W. J. (2006). Mobility of synaptic vesicles in different pools in resting and stimulated frog motor nerve terminals. Neuron 51, 317-325.

Geppert, M., Ullrich, B., Green, D. G., Takei, K., Daniels, L., De Camilli, P., Sudhof, T. C., and Hammer, R. E. (1994). Synaptic targeting domains of synapsin I revealed by transgenic expression in photoreceptor cells. EMBO J. 13, 3720-3727.

\section{ACKNOWLEDGMENTS}

The research leading to these results has received funding from the European Union Seventh Framework Programme under grant agreement no. HEALTH-F2-2009-242167 ("SynSys" project), Swedish Research Council (grants: 13473, 20587, 21627), Linné Center DBRM, Wallenbergs Stifrtelser, ESF: DFG HA 2686 6-1 and VR 90664601 . We would like to thank Dr. V. Lucic for the original image for the Figure 1E.

Gitler, D., Takagishi, Y., Feng, J., Ren, Y., Rodriguiz, R. M., Wetsel, W. C., Greengard, P., and Augustine, G. J. (2004). Different presynaptic roles of synapsins at excitatory and inhibitory synapses. J. Neurosci. 24, 11368-11380.

Godenschwege, T. A., Reisch, D., Diegelmann, S., Eberle, K., Funk, N., Heisenberg, M., Hoppe, V., Hoppe, J., Klagges, B. R., Martin, J. R., Nikitina, E. A., Putz, G., Reifegerste, R., Reisch, N., Rister, J., Schaupp, M., Scholz, H., Schwarzel, M., Werner, U., Zars, T. D., Buchner, S., and Buchner, E. (2004). Flies lacking all synapsins are unexpectedly healthy but are impaired in complex behaviour. Eur. J. Neurosci. 20, 611-622.

Greengard, P., Valtorta, F., Czernik, A J., and Benfenati, F. (1993). Synaptic vesicle phosphoproteins and regulation of synaptic function. Science 259, 780-785.

Gustafsson, J. S., Birinyi, A., Crum, J., Ellisman, M., Brodin, L., and Shupliakov, O. (2002). Ultrastructural organization of lamprey reticulospinal synapses in three dimensions. J. Comp. Neurol. 450, 167-182.

Haffner, C., Takei, K., Chen, H., Ringstad, N., Hudson, A., Butler, M. H., Salcini, A. E., Di Fiore, P. P., and De Camilli, P. (1997). Synaptojanin 1: localization on coated endocytic intermediates in nerve terminals and interaction of its $170 \mathrm{kDa}$ isoform with Eps15. FEBS Lett. 419, 175-180.

Henkel, A. W., Simpson, L. L., Ridge, R. M., and Betz, W. J. (1996). Synaptic vesicle movements monitored by fluorescence recovery after photobleaching in nerve terminals stained with FM1-43. J. Neurosci. 16, 3960-3967.

Hirokawa, N., Sobue, K., Kanda, K., Harada, A., and Yorifuji, H. (1989). The cytoskeletal architecture of the presynaptic terminal and molecular structure of synapsin 1. J. Cell Biol. 108, 111-126.

Jakobsson, J., Gad, H., Andersson, F., Low, P., Shupliakov, O., and Brodin, L. (2008). Role of epsin 1 in synaptic vesicle endocytosis. Proc. Natl. Acad. Sci. U.S.A. 105, 6445-6450.
Koh, T. W., Korolchuk, V. I., Wairkar, Y. P., Jiao, W., Evergren, E., Pan, H., Zhou, Y., Venken, K. J., Shupliakov, O., Robinson, I. M., O’Kane, C. J., and Bellen, H. J. (2007). Eps15 and Dap160 control synaptic vesicle membrane retrieval and synapse development. $J$ Cell Biol. 178, 309-322.

Koh, T. W., Verstreken, P., and Bellen, H. J. (2004). Dap160/intersectin acts as a stabilizing scaffold required for synaptic development and vesicle endocytosis. Neuron 43, 193-205.

Kraszewski, K., Daniell, L., Mundigl, O., and De Camilli, P. (1996). Mobility of synaptic vesicles in nerve endings monitored by recovery from photobleaching of synaptic vesicleassociated fluorescence. J. Neurosci. 16, 5905-5913.

Lenzi, D., Runyeon, J. W., Crum, J., Ellisman, M. H., and Roberts, W. M. (1999). Synaptic vesicle populations in saccular hair cells reconstructed by electron tomography. J. Neurosci. 19, 119-132.

Li, L, Chin, L. S., Shupliakov, O., Brodin, L., Sihra, T. S., Hvalby, O., Jensen, V., Zheng, D., McNamara, J. O., Greengard, P., and Andersen, P. (1995). Impairment of synaptic vesicle clustering and of synaptic transmission, and increased seizure propensity, in synapsin I-deficient mice. Proc. Natl. Acad. Sci. U.S.A. 92, 9235-9239.

Monaldi, I., Vassalli, M., Bachi, A., Giovedi, S., Millo, E., Valtorta, F., Raiteri, R., Benfenati, F., and Fassio, A. (2009). The highly conserved synapsin domain E mediates synapsin dimerization and phospholipid vesicle clustering. Biochem. J. 426, 55-64.

Parsons, T. D., and Sterling, P. (2003). Synaptic ribbon. Conveyor belt or safety belt? Neuron 37, 379-382.

Pieribone, V. A., Shupliakov, O., Brodin, L., Hilfiker-Rothenfluh, S., Czernik, A. J., and Greengard, P. (1995). Distinct pools of synaptic vesicles in neurotransmitter release. Nature 375, 493-497.

Richards, D. A., Guatimosim, C., Rizzoli, S. O., and Betz, W. J. (2003). Synaptic vesicle pools at the frog neuromuscular junction. Neuron 39, 529-541. 
Rizzoli, S. O., and Betz, W. J. (2005). Synaptic vesicle pools. Nat. Rev. Neurosci. 6, 57-69.

Ryan, T. A., Li, L., Chin, L. S., Greengard, P., and Smith, S. J. (1996). Synaptic vesicle recycling in synapsin I knock-out mice. J. Cell Biol. 134, 1219-1227.

Sankaranarayanan, S., Atluri, P. P., and Ryan, T.A. (2003). Actin has a molecular scaffolding, not propulsive, role in presynaptic function. Nat. Neurosci. 6, 127-135.

Schikorski, T., and Stevens, C. F. (2001). Morphological correlates of functionally defined synaptic vesicle populations. Nat. Neurosci. 4, 391-395.

Shupliakov, O. (2009). The synaptic vesicle cluster: a source of endocytic proteins during neurotransmitter release. Neuroscience 158, 204-210.

Shupliakov, O., Bloom, O., Gustafsson, J. S., Kjaerulff, O., Low, P., Tomilin, N., Pieribone, V. A., Greengard, P., and
Brodin, L. (2002). Impaired recycling of synaptic vesicles after acute perturbation of the presynaptic actin cytoskeleton. Proc. Natl. Acad. Sci. U.S.A. 99, 14476-14481.

Siksou, L., Rostaing, P., Lechaire, J. P., Boudier, T., Ohtsuka, T., Fejtova, A., Kao, H.T., Greengard, P., Gundelfinger, E. D., Triller, A., and Marty, S. (2007). Three-dimensional architecture of presynaptic terminal cytomatrix. J. Neurosci. 27, 6868-6877.

Staras, K., Branco, T., Burden, J. J., Pozo, K., Darcy, K., Marra, V., Ratnayaka, A., and Goda, Y. (2010). A vesicle superpool spans multiple presynaptic terminals in hippocampal neurons. Neuron 66 , 37-44.

Südhof, T. C. (2000). The synaptic vesicle cycle revisited. Neuron 28, 317-320.

Südhof, T. C. (2004). The synaptic vesicle cycle. Annu. Rev. Neurosci. 27, 509-547.

Tao-Cheng, J. H. (2006). Activity-related redistribution of presynaptic proteins at the active zone. Neuroscience 141 1217-1224.

Tao-Cheng, J. H., Dosemeci, A., Winters, C. A., and Reese, T. S. (2006). Changes in the distribution of calcium calmodulin-dependent protein kinase II at the presynaptic bouton after depolarization. Brain Cell Biol. 35, 117-124.

Terada, S., Tsujimoto, T., Takei, Y., Takahashi, T., and Hirokawa, N. (1999). Impairment of inhibitory synaptic transmission in mice lacking synapsin I. J. Cell Biol. 145, 1039-1048.

Usukura, J., and Yamada, E. (1987). Ultrastructure of the synaptic ribbons in photoreceptor cells of Rana catesbeiana revealed by freeze-etching and freeze-substitution. Cell Tissue Res. 247, 483-488.

Valtorta, F., Greengard, P., Fesce, R., Chieregatti, E., and Benfenati,F. (1992). Effects of the neuronal phosphoprotein synapsin I on actin polymerization. I. Evidence for a phosphorylation- dependent nucleating effect. J. Biol. Chem. 267, 11281-11288.

Conflict of Interest Statement: The authors declare that the research was conducted in the absence of any commercial or financial relationships that could be construed as a potential conflict of interest.

Received: 21 June 2010; paper pending published:22July 2010; accepted:23 August 2010; published online: 15 September 2010.

Citation: Pechstein A and Shupliakov O (2010) Taking a back seat: synaptic vesicle clustering in presynaptic terminals. Front. Syn. Neurosci. 2:143 doi:10.3389/ fnsyn.2010.00143

Copyright $\odot 2010$ Pechstein and Shupliakov. This is an open-access article subject to an exclusive license agreement between the authors and the Frontiers Research Foundation, which permits unrestricted use, distribution, and reproduction in any medium, provided the original authors and source are credited. 